\title{
ON THE FINITE IMAGES OF SOME ONE-RELATOR GROUPS
}

\author{
D. MOLDAVANSKI AND N. SIBYAKOVA
}

(Communicated by Ronald Solomon)

\begin{abstract}
It is shown that the group $G=\left\langle a, b ; a^{-1} b a=b^{k}\right\rangle \quad(k \neq 0)$ is determined in the class of all residually finite one-relator groups by the set of its finite images.
\end{abstract}

Let $\mathscr{F}(G)$ denote the set of all finite homomorphic images of a group $G$. Also let $G_{k}(m)$ be the group with presentation

$$
\left\langle a, b ; a^{-1} b a=b^{k}, b^{m}=1\right\rangle,
$$

where the integers $k \neq 0$ and $m>0$ are coprime.

G. Baumslag has noted in [1] that there exist integers $k, l, m$ (e.g. $m=$ $25, k=6, l=11)$ such that the groups $G_{k}(m)$ and $G_{l}(m)$ are not isomorphic but $\mathscr{F}\left(G_{k}(m)\right)=\mathscr{F}\left(G_{l}(m)\right)$. In fact, the situation is entirely described by the following statement: $\mathscr{F}\left(G_{k}(m)\right)=\mathscr{F}\left(G_{l}(m)\right)$ if and only if the cosets $k+m \mathbb{Z}$ and $l+m \mathbb{Z}$ generate the same cyclic subgroups of the group $\mathbb{Z}_{m}^{*}$, the multiplicative group of integers relatively prime to $m$ in the ring $\mathbb{Z}_{m}$ of integers modulo $m$. Moreover, $G_{k}(m) \simeq G_{l}(m)$ if and only if $k+m \mathbb{Z}=(l+m \mathbb{Z})^{ \pm 1}$.

The "if" part of the first assertion as well as the second assertion was proved in [1], and the "only if" part of the first assertion was proved in [2].

Any group $G_{k}(m)$ is a factor group of the one-relator group

$$
G_{k}=\left\langle a, b ; a^{-1} b a=b^{k}\right\rangle \quad(k \neq 0),
$$

and by contrast with the above result we have

Theorem 1. $\mathscr{F}\left(G_{k}\right)=\mathscr{F}\left(G_{l}\right)$ if and only if $k=l$.

Proof. For any integers $r>0$ and $s>0$ satisfying the condition $k^{r} \equiv 1(\bmod s)$ we define $G_{k}(r, s)$ to be the group with presentation

$$
\left\langle a, b ; a^{-1} b a=b^{k}, a^{r}=1, b^{s}=1\right\rangle .
$$

It is well known that $G_{k}(r, s)$ is a finite metacyclic group of order $r s$. Any element of $G_{k}(r, s)$ is uniquely representable in the form $a^{\alpha} b^{\beta}$ where $0 \leq$ $\alpha<r, 0 \leq \beta<s$. We notice also that the commutator subgroup of the group $G_{k}(r, s)$ is the cyclic subgroup generated by the element $b^{k-1}$.

Received by the editors May 5, 1993 and, in revised form, November 22, 1993.

1991 Mathematics Subject Classification. Primary 20F05; Secondary 20E26.

The work of the first author was supported by a grant of the High School Committee of Russia. 
Let $\varphi$ be a homomorphism of the group $G_{k}$ into some finite group, and let $r$ and $s$ be the respective orders of the elements $a \varphi$ and $b \varphi$. The relation $a^{-\alpha} b a^{\alpha}=b^{k^{\alpha}}$ which holds in the group $G_{k}$ for all $\alpha \geq 0$ shows that the integers $r$ and $s$ satisfy the condition $k^{r} \equiv 1(\bmod s)$. This implies that any homomorphism of the group $G_{k}$ into a finite group passes through some group $G_{k}(r, s)$.

In particular we see that if $k \neq 1$, then the group $G_{k}$ has a non-abelian finite image. Therefore if $\mathscr{F}\left(G_{k}\right)=\mathscr{F}\left(G_{l}\right)$, then $k=1$ if and only if $l=1$, and we can assume in what follows that the numbers $k$ and $l$ are not equal to 1 .

Now we prove two lemmas.

Lemma 1. If $\mathscr{F}\left(G_{k}\right) \subseteq \mathscr{F}\left(G_{l}\right)$, then for every prime $p$ and for any integer $t \geq 0$ $p^{t} \mid k-1$ implies $p^{t} \mid l-1$.

(Here and everywhere below the notation $r \mid s$ will mean that the integer $r$ divides the integer $s .(r, s)$ denotes the greatest common divisor of $r$ and $s$.)

To prove this assertion let us write the integers $k$ and $l$ in the form

$$
k=1+p^{r} u, \quad l=1+p^{s} v,
$$

where $r \geq 0, s \geq 0$, and $(u, p)=(v, p)=1$. If $r>0$, then $k^{p} \equiv 1\left(\bmod p^{r+1}\right)$ and we may consider the group $G_{k}\left(p, p^{r+1}\right)$. This group must be an image of the group $G_{l}$ and, therefore, of some group $G_{l}\left(p^{m}, p^{n}\right)$. Since the group $G_{k}\left(p, p^{r+1}\right)$ is non-abelian, $n>s$. Hence the commutator subgroup of $G_{l}\left(p^{m}, p^{n}\right)$, generated by the element $b^{p^{s} v}$, has the order $p^{s}=\left(p^{s} v, p^{n}\right)$. Since the order of the commutator subgroup of the group $G_{k}\left(p, p^{r+1}\right)$ is equal to $p^{r}$, we must have $s \geq r$.

Lemma 2. Let $\mathscr{F}\left(G_{k}\right) \subseteq \mathscr{F}\left(G_{l}\right)$. Then every prime divisor of $l$ divides $k$.

Proof. Suppose that there is a prime number $p$ such that $p \mid l$ and $p \nmid k$. Since $p \nmid l-1$, by Lemma $1, p \nmid k-1$. Therefore the group $G_{k}(p-1, p)$ is not abelian and its commutator subgroup is of order $p$. Any epimorphism $\varphi$ of $G_{l}$ onto $G_{k}(p-1, p)$ passes through some group $G_{l}(r, s)$ where $s$ is the order of $b \varphi$, and therefore $(s, l)=1$. Consequently, the commutator subgroup of $G_{k}(p-1, p)$ is generated by the element $\left(b^{l-1}\right) \varphi$. Hence the element $(b \varphi)^{l-1}$ is of order $p$, but this is impossible since $p \mid l$ and $(s, l)=1$.

Suppose now that for some integers $k$ and $l$ the equality $\mathscr{F}\left(G_{k}\right)=\mathscr{F}\left(G_{l}\right)$ holds. It follows from Lemma 1 that the integers $k-1$ and $l-1$ are distinguished at most by sign. Therefore if $k \neq l$ one must have $k+l=2$. Let $k=2^{r} k_{1}$ and $l=2^{s} l_{1}$ where $r \geq 0, s \geq 0$, and $k_{1}$ and $l_{1}$ are odd. Lemma 2 implies that the integers $k$ and $l$ have the same prime divisors, and therefore, since $k+l=2, k_{1}, l_{1}= \pm 1$. If we assume, without loss of generality, that $r \leq s$, then the equality $2^{r}\left(k_{1}+2^{s-r} l_{1}\right)=2$ implies $r=0$ or $r=1$. If $r=0$, then $s=r$ because the integer $k_{1}+2^{s-r} l_{1}$ must be even. Hence $k_{1}=l_{1}=1$, and so $k=l$. Let $r=1$. Then $k_{1}+2^{s-1} l_{1}=1$ and therefore $k_{1}=-1, l_{1}=1$, and $s=2$. Thus in this case $k=-2, l=4$. Consequently, it remains to show that $\mathscr{F}\left(G_{-2}\right) \neq \mathscr{F}\left(G_{4}\right)$.

To do this, we shall show that if the elements $f$ and $g$ of the group $G_{4}(2,5)$ satisfy the condition $f^{-1} g f=g^{-2}$, then $g=1$. 
Let these elements be written in the form

$$
f=a^{\alpha} b^{\beta}, \quad g=a^{\gamma} b^{\delta} \quad(0 \leq \alpha, \gamma<2,0 \leq \beta, \delta<5) .
$$

By factorization of the group $G_{4}(2,5)$ by the subgroup generated by the element $b$ the equality $f^{-1} g f=g^{-2}$ becomes $a^{3 \gamma}=1$, and we must have $\gamma=0$. Therefore $f^{-1} g f=b^{-\beta} a^{-\alpha} b^{\delta} a^{\alpha} b^{\beta}=b^{\delta 4^{\alpha}}$ and $g^{-2}=b^{-2 \delta}$. Thus

$$
\delta\left(4^{\alpha}+2\right) \equiv 0 \quad(\bmod 5),
$$

and it follows that $\delta=0$. The proof of Theorem 1 is completed.

It is worthwhile to make some additional remarks. At first, what can one say about a one-relator group $G$ such that $\mathscr{F}(G)=\mathscr{F}\left(G_{k}\right)$ ? In the general case the answer is unknown, but the question can be easily answered when $G$ is residually finite.

Corollary. If $G$ is a residually finite one-relator group and if for some integer $k, \mathscr{F}(G)=\mathscr{F}\left(G_{k}\right)$, then $G \simeq G_{k}$.

To prove this, it is enough to notice that the group $G_{k}$ and therefore all groups in $\mathscr{F}\left(G_{k}\right)$ are metabelian. Consequently, $G$ is metabelian since $G$ is a subdirect product of the family $\mathscr{F}(G)=\mathscr{F}\left(G_{k}\right)$. Since $G$ is not cyclic, by [3], $G$ is isomorphic to some group $G_{l}$. From Theorem 1 it follows that $l=k$.

Following [4], we denote by $\sigma G$ the sequence whose $n$th term, $\sigma_{n} G$, is the number of subgroups of index $n$ of a group $G$. It turns out that for any finitely generated groups $G$ and $H, \mathscr{F}(G)=\mathscr{F}(H)$ implies $\sigma G=\sigma H$.

Indeed, if $N$ is a normal subgroup of $G$, then for any number $n \geq 1$ we have $\sigma_{n} G \geq \sigma_{n}(G / N)$, equality holding if and only if all subgroups of index $n$ of $G$ contain $N$. Since the group $G$ is finitely generated, it contains only a finite number of subgroups of index $n$, and therefore their intersection $U_{n}$ is a subgroup of finite index of $G$. Consequently the quotient group $G / U_{n}$ is isomorphic to some $H / N$, and therefore

$$
\sigma_{n} H \geq \sigma_{n}(H / N)=\sigma_{n}\left(G / U_{n}\right)=\sigma_{n} G .
$$

The next result and Theorem 1 show in particular that the converse of the above statement is false.

Theorem 2. For any integer $n \geq 1, \sigma_{n}\left(G_{k}\right)$ is the sum of all positive divisors of $n$ that are coprime with $k$, and $\sigma_{n}\left(G_{k}(m)\right)$ is the sum of all positive common divisors of $m$ and $n$.

We give a sketch of the proof of Theorem 2. Let $H(p, q, r)$ be the subgroup of $G_{k}$ generated by two elements $a^{p} b^{r}$ and $b^{q}$, where $p>0, q>0$, and $q$ is coprime with $k$. The following assertions can be easily verified and produce the required proof:

(1) Every subgroup of finite index of $G_{k}$ coincides with some $H(p, q, r)$.

(2) $\left[G_{k}: H(p, q, r)\right]=p q$.

(3) $H\left(p_{1}, q_{1}, r_{1}\right)=H\left(p_{2}, q_{2}, r_{2}\right)$ if and only if $p_{1}=p_{2}, q_{1}=q_{2}$, and $r_{1} \equiv r_{2}\left(\bmod q_{1}\right)$.

(4) The subgroup $H(p, q, r)$ contains the normal closure in $G_{k}$ of the element $b^{m}$ if and only if $q$ divides $m$.

It can also be shown that the subgroup $H(p, q, r)$ of the group $G_{k}$ is isomorphic to the group $G_{l}$, where $l=k^{p}$. Thus Theorem 1 shows the existence 
of two groups, $G$ and $H$, having isomorphic normal subgroups $A$ and $B$ of finite index such that $G / A \simeq H / B$ and $\mathscr{F}(G) \neq \mathscr{F}(H)$.

Finally, we want to mention the question of the existence of an infinite family of one-relator groups which are not isomorphic in pairs and have the same finite images. One example of such a family is prompted by a note of G. Baumslag [5]. Let $H_{m}$ be the group with presentation

$$
\left\langle a, b ; a^{-m} b^{-1} a^{m} b a^{-m} b a^{m}=b^{2}\right\rangle \quad(m>0) .
$$

It is shown in [5] that $\mathscr{F}\left(H_{1}\right)$ coincides with $\mathscr{F}(\mathbb{Z})$, the set of all finite cyclic groups, and the same arguments show the validity of the equality $\mathscr{F}\left(H_{m}\right)=$ $\mathscr{F}(\mathbb{Z})$ for any $m>0$. The normal closure $N_{m}$ of the element $b$ in $H_{m}$ is the unique invariant subgroup of $H_{m}$ whose quotient is infinite cyclic. The group $N_{m}$ is the free product of $m$ freely indecomposable groups. Therefore the groups $H_{m}$ and $H_{n}$ are not isomorphic if $m \neq n$. Nevertheless the groups $H_{m}$ are not residually finite. The problem of the existence of an analogous family of residually finite one-relator groups is still open.

\section{ACKNOWLEDGMENT}

The authors are very grateful to the referee for pointing to [2] and for comments which have promoted the simplification of the original proof of Theorem 1 .

\section{REFERENCES}

1. G. Baumslag, Residually finite groups with the same finite images, Compositio Math. 29 (1974), 249-252.

2. M. Burrow and A. Steinberg, On a result of G. Baumslag, Compositio Math. 71 (1989), 241-245.

3. W. Magnus, Uber diskontinuierliche gruppen mit einer definieren den relation (der Freiheitssatz), J. Reine Angew. Math. 163 (1930), 141-165.

4. G. Baumslag, Some problems on one-relator groups, Proc. Second Internat. Conf. Theory of Groups, Canberra, 1973, pp. 75-81.

5. $\longrightarrow$ A non-cyclic one-relator group all of whose finite quotients are cyclic, J. Austral. Math. Soc. 10 (1969), 497-498.

Department of Mathematics, Ivanovo State University, Ivanovo, 153002, Russia

E-mail address: svi@ivgu.ivanovo.su 